\title{
Evaluation of Add/Drop Flexibility Requirements in Future SDM-enabled ROADMs
}

\author{
Jordi Perelló ${ }^{\text {* }}$, Joan M. Gené ${ }^{1}$, Mirosław Klinkowski², Jaume Comellas ${ }^{1}$, and Salvatore Spadaro ${ }^{1}$ \\ ${ }^{1}$ Universitat Politècnica de Catalunya (UPC) - BarcelonaTech, Barcelona, Spain \\ ${ }^{2}$ National Institute of Telecommunications, Warsaw, Poland \\ *E-mail: perello@ac.upc.edu
}

\begin{abstract}
In this paper, we evaluate five different architectures for implementing the Add/Drop module of future Spatial Division Multiplexing (SDM)-enabled Re-configurable Optical Add \& Drop Multiplexers (ROADMs) with different degrees of flexibility, from a completely static transponder to spatial channel assignment to a fullyflexible transceiver to fibre and spatial channel assignment. The performance of the proposed architectures is compared in a single SDM-enabled ROADM scenario, where local as well as bypass traffic is injected, supported by spectral super-channels at diverse bit-rates and employing different modulation formats. The obtained results illustrate the additional benefits attainable by more flexible Add/Drop architectures versus their increased hardware complexity.
\end{abstract}

Keywords: Flex-Grid, SDM, ROADM, Add/Drop.

\section{INTRODUCTION}

Elastic Optical Networks (EONs) implementing Flex-Grid and Spatial Division Multiplexing (SDM) technologies [1] have emerged as strong candidates to realize next-generation optical network infrastructures, able to scale their capacity far beyond the nonlinear Shannon limit of standard Single Mode Fibres (SMFs). In the short-term, Flex-Grid/SDM optical networks can be realized employing SMF Bundles (SMFBs) per link, multiplying the Flex-Grid network capacity by the number of SMFs per bundle. Conversely, mid- and long-term Flex-Grid/SDM optical network realizations are envisioned to rely on advanced optical fibre technologies like Multi-Core Fibres (MCFs), Few-Mode Fibres (FMFs) or Few-Mode MCFs (FM-MCFs).

So far, many works have proposed and evaluated different ultra-high bit-rate super-channel configurations (e.g., see [2]), like spectral super-channels, with their composing sub-channels arranged across the spectral domain, or spatial ones, with their sub-channels arranged across the spatial domain (over the same central frequency). Spectral-spatial super-channels, arranging their sub-channels across both spectral and spatial domains, have also been studied. These super-channels can be affected by crosstalk-related impairments when employing MCF, FMF and FM-MCF advanced optical fibre technologies [3]. Therefore, new transmission reach estimation models become mandatory, as the ones proposed in [4].

Some works have also concentrated on proposing novel SDM-ROADM architectures, ranging from fullyflexible but expensive ones, able to switch any spectral portion from any input fibre/core/mode to any output one (a technique called Independent Switching - InS), to more cost-effective ones realizing the Joint Switching (JoS) technique, applicable to spatial super-channels only [5]. Another cost-effective SDM-ROADM architecture supporting spectral and spatial super-channels is the one performing InS without lane change [6], which trades fibre/core/mode continuity for a simpler SDM-ROADM architecture, comparable to that realizing JoS, offering a spectral efficiency close to that obtained with pure InS [7].

However, the Add/Drop module of the SDM-ROADM, inter-connecting the available transceivers with the output spatial channels have remained uncovered by most related works, assuming that it offers full flexibility, which can entail high technological complexity and associated costs. Therefore, this paper proposes five different architectures for the Add/Drop module of SDM-ROADMs, ranging from completely static to fullyflexible ones, aiming to evaluate whether (or not) less flexible but potentially cheaper architectures can still match the performance of the fully-flexible one assumed in most related works.

\section{ADD/DROP MODULE ARCHITECTURES FOR SDM-ROADMS}

Five different architectures for the Add/Drop module of future SDM-ROADMs are proposed and evaluated in this work, called Static-Tp, Flex-Tp2C, Flex-Tp2F\&C, Flex-Tc2C and Flex-Tc2F\&C, being the latter four depicted in Figure 1. Static-Tp refers to a static transponder to spatial channel assignment, containing each transponder an array (or a pool) of $T$ transceivers, connected to the same output spatial channel in a fixed static way. These transceivers can only support connections originated in the SDM-ROADM, departing through that specific output channel (bidirectional connections would follow the reverse path). In general, denoting as $D, C$ and $S$ the SDM-ROADM degree, number of spatial channels per SDM fibre and total number of transponders per SDM-ROADM direction, respectively, the total number of available transceivers in the Add/Drop module is $D \cdot S \cdot T$, if one transponder is connected to each output spatial channel (i.e., $S=C$ ). 
Figure 1.a depicts the alternative Flex-Tp2C architecture, where some flexibility in the Add/Drop module (dashed box) is added by means of $1 x C$ switches and Sxl combiners, interconnecting transponders with any of the $C$ spatial channels of the same output SDM fibre. It is worth mentioning that in Flex-Tp2C, once a transponder is interconnected with a specific output spatial channel, its entire transceiver array can only transmit optical signals to that spatial channel. Figure 1.b illustrates an enhanced version of this architecture, called Flex-Tp2F\&C, where transponders are not dedicated to specific output SDM fibres (as in Static-Tp or Flex-Tp2C), but can be interconnected with any spatial channel of any output SDM fibre. This is achieved with the introduction of new $1 x D$ switches, additional $1 x C$ switches and higher port-count $(D x S) x 1$ combiners. But again, once a transponder is interconnected with a specific output spatial channel, its entire transceiver array can only transmit to that spatial channel. Figures 1.c and 1.d present Add/Drop module architectures where individual transceivers can be interconnected with output spatial channels, thus gaining superior flexibility. In Figure 1.c the Flex-Tc2C architecture is presented, where an array of $S x T$ transceivers is assigned to each SDM-ROADM direction. And Figure 1.d shows the fully-flexible Flex-Tc2F\&C architecture, where a single array of $D x S x T$ transceivers exist, so that they can be individually interconnected to any output spatial channel. However, both architectures entail higher hardware complexity (and cost) in terms of additional $1 x C$ switches and higher port-count combiners. Note that all 5 architectures assume $D \cdot S \cdot T$ transceivers in total at the SDM-ROADM.

\section{TSSA HEURISTIC}

In this section, we present the Transceiver, Spatial channel and Spectrum Assignment (TSSA) heuristic that we employ to allocate new lightpaths when adopting each one of the proposed Add/Drop module architectures, in order to evaluate their performance in section 4. It is worth mentioning that this heuristic, described in Algorithm 1, is only applicable to local Add/Drop lightpaths originating/terminating at the SDM-ROADM.

The TSSA heuristic described in Algorithm 1 receives as input parameters the Add/Drop module architecture type, output SDM fibre to which the new local Add/Drop lightpath has to be setup $(f)$, as well as the number of required transceivers $\left(N_{\text {transc }}\right)$ and FSs $\left(N_{\mathrm{fs}}\right)$. Lines 1-9 describe the procedure applied to Static-Tp, Flex-Tc2C and Flex-Tc2F\&C architecture types. In these cases, the heuristic starts exploring the spatial channels in output fibre $f$ sequentially, from 1 to $C$. In each of these channels, it seeks for a spectral slice of $N_{\mathrm{fs}}$ contiguously available FSs, in a first-fit fashion. If such a slice is found in spatial channel $c$, it then starts analysing if $N_{\text {transc }}$ are available in such transceiver arrays able to send traffic to it. For example, in the Static-Tp architecture, there is only one transceiver array associated to every spatial channel in every output SDM fibre, whereas in Flex-Tc2C, transceiver availability would be sought in the transceiver array associated to output fibre $f$. If $N_{\text {transc }}$ are available, they are reserved together with the spectral slice found before and the lightpath is considered as served. Otherwise, the lightpath is dropped (line 28).

For architectures Flex-Tp2C and Flex-Tp2F\&C (lines 10-27) the procedure is similar. However, only transceivers already in use sending traffic to the same spatial channel where an available spectral slice of $N_{\mathrm{fs}}$ contiguous FSs has been found are initially explored, to find if the required $N_{\text {transc }}$ transceivers are still available there. If so, the available spectral resources and transceivers found are reserved and the lightpath is considered as served. Otherwise, a random spatial channel index $c$ is obtained and spatial channels are explored from then on, in a round-robin fashion. As IDLE transceiver arrays (i.e., transponders) will be explored this time, this avoids inter-connecting too many transponders with spatial channels with lower indexes (their entire transceiver arrays would only be able to send traffic over them). If an available spectral slice of $N_{\mathrm{fs}}$ contiguous FSs is found in spatial channel $c$, IDLE transceiver arrays (i.e., transponders) are explored. If $N_{\text {transc }}$ are available, they are reserved together with the spectral slice found and the lightpath is served. Conversely, it is dropped (line 28).

To allocate incoming bypass lightpaths, which are also considered in the evaluation, a simpler first-fit spatial channel and spectrum assignment heuristic is applied, given their input and output SDM fibre to/from the SDMROADM (on their route from source to destination). As the SDM-ROADM is assumed to perform InS without lane change, spatial channel continuity must be enforced from input to output SDM fibres, besides the spectrum contiguity constraint imposed by the Flex-Grid transmission technology. Then, assuming spatial channels in 
SDM fibres ordered from 1 to $C$, they are explored in that same order, in both input and output SDM fibres together. Given a pair of spatial channels (with the same index), it is checked, in a first-fit fashion, whether $N_{\mathrm{fs}}$ frequency slots are contiguously and continuously available in both of them, being $N_{\mathrm{fs}}$ the number of FSs required by the new incoming bypass lightpath request. If available, the FSs found are reserved in both input and output SDM fibres and the bypass lightpath is considered as served. Conversely, if no $N_{\mathrm{fs}}$ FSs are contiguously and continuously available in any pair of spatial channels, the bypass lightpath is considered as blocked.

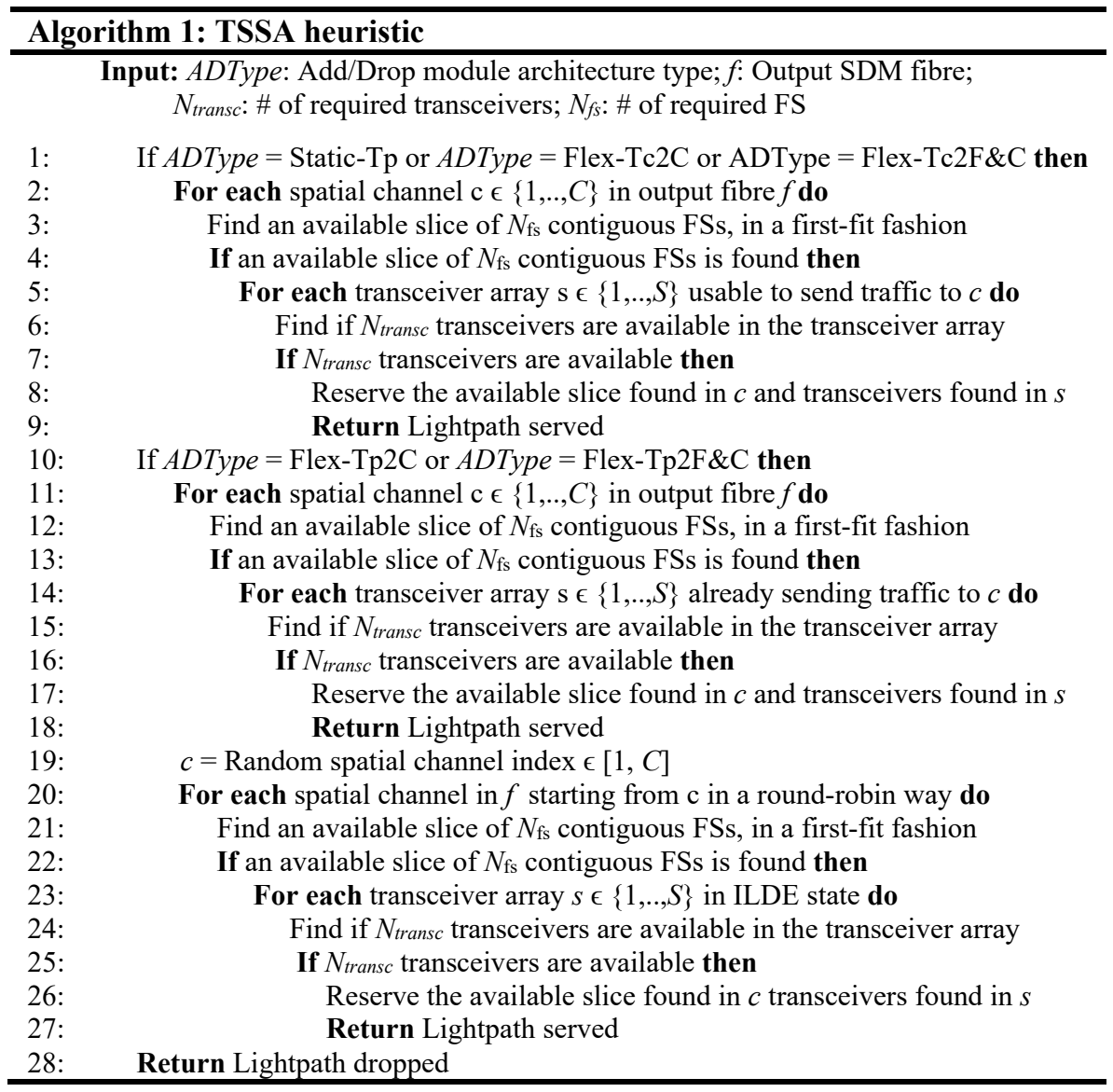

\section{NUMERICAL RESULTS AND COMPARISON}

In order to evaluate and compare the proposed architectures, simulations are conducted in a single SDMROADM scenario using an ad-hoc Java-based simulator. There, connection requests arrive following a Poisson process, with negative exponentially distributed inter-arrival and holding times, with mean values equal to IAT and HT. The SDM-ROADM is considered of degree 5 with 7 spatial channels per SDM fibre (i.e., $D=5, C=7$ ). The 4-THz C-Band is available in all spatial channels, discretized into $320 \mathrm{FSs}$ of $12.5 \mathrm{GHz}$ width.

Bypass and local Add/Drop requests are offered, so that the $\%$ of each request type is configured as an input parameter. When a new request arrives, its bit-rate is firstly decided, assuming a $100 \mathrm{~Gb} / \mathrm{s}(40 \%)-400 \mathrm{~Gb} / \mathrm{s}$ $(30 \%)-1000 \mathrm{~Gb} / \mathrm{s}(30 \%)$ traffic profile. Moreover, the modulation format used by that connection request is randomly chosen among PM-QPSK, PM-16-QAM and PM-64-QAM, with probabilities 0.5, 0.25 and 0.25, respectively. If the request type is bypass, its input/output SDM fibres in the SDM-ROADM are randomly selected among the available ones, which must be different. Conversely, when the request type is local Add/Drop, only an output SDM fibre is randomly selected. Then, its number of required FSs is computed as $\mathrm{N}_{\mathrm{fs}}$ $=$ ceil $\left[\left(\right.\right.$ BitRate/Mod $\left.\left.\mathrm{Mff}_{\mathrm{e}}+\mathrm{GB}\right) / \mathrm{FS}_{\text {width }}\right]$, being BitRate, Mod $\mathrm{eff} \mathrm{GB}$ and $\mathrm{FS}_{\text {width }}$ the bit-rate of the request, efficiency of the employed modulation format $(4,8$ or $12 \mathrm{~b} / \mathrm{s} / \mathrm{Hz})$, width of the guard-bands between adjacent spectral super-channels $(12.5 \mathrm{GHz})$ and width of the spectrum FSs $(12.5 \mathrm{GHz})$. Finally, the number of required transceivers is also computed for local Add/Drop requests as $\mathrm{N}_{\text {transc }}=$ ceil [BitRate/(Mod eff $^{*}$ Mod $\left.\mathrm{d}_{\text {baud }}\right)$ ], being Mod $_{\text {baud }}$ the baud-rate of the optical modulators (32 Gbaud).

The offered load (HT/IAT) is set to 160 , which leads to Bandwidth Blocking Probability (BBP) around $1 \%$ with infinite transceivers available at the SDM-ROADM, no matter the Add/Drop module architecture, when $50 \%$ of requests are bypass and the remainder $50 \%$ local Add/Drop ones (benchmark scenario). Then, the number of available transceivers at the SDM-ROADM is ranged from $280(\mathrm{~T}=8)$ to $560(\mathrm{~T}=16)$. Figure 2 shows the obtained results for 3 different scenarios. 

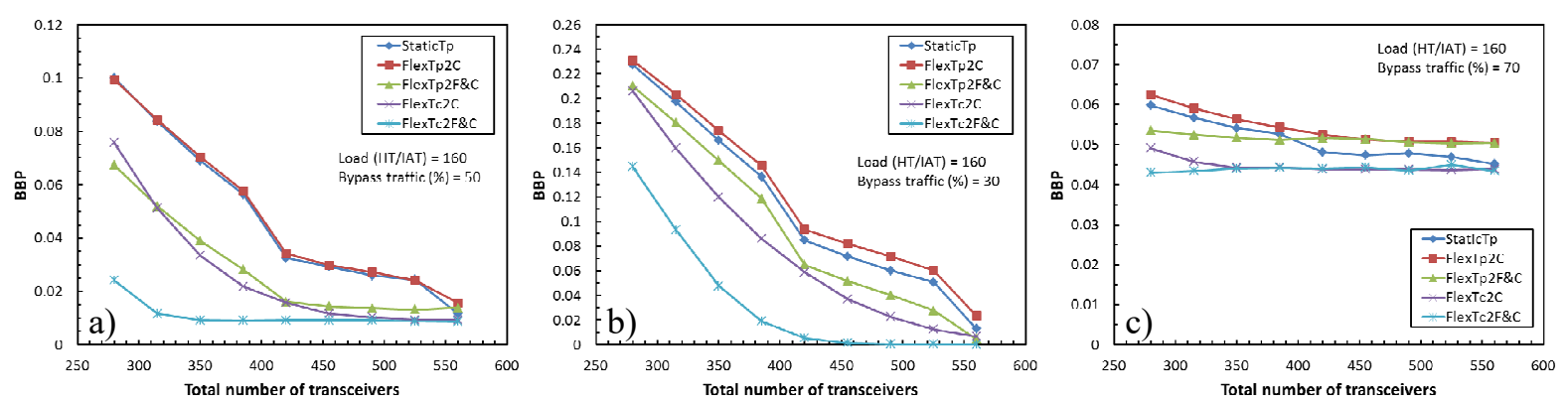

Figure 2. BBP versus total number of available transceivers at the SDM-ROADM under an offered load of 160, with $50 \%$ (a), 30\% (b) and 70\% (c) of bypass connection requests, while the remainder are local Add/Drop.

As seen in Figure 2.a, when bypass traffic is $50 \%$, the fully-flexible Flex-Tc2F\&C architecture behaves the best, still yielding $\mathrm{BBP} \approx 1 \%$ with 310 transceivers in total at the SDM-ROADM. The intermediate (in terms of flexibility and complexity) Flex-Tp2F\&C and Flex-Tc2C architectures perform quite similar, requiring around 450 transceivers at the SDM-ROADM, so as to yield $\mathrm{BBP} \approx 1 \%$. Finally, Static-Tp and Flex-Tp2C provide the poorest performance, requiring both around 560 transceivers at the SDM-ROADM to provide BBP $\approx 1 \%$. Hence, the increased complexity of Flex-Tp2C vs. Static-Tp does not seem to pay off.

Figures 2.b and 2.c illustrate the same results, but modifying the $\%$ of bypass connection requests to $30 \%$ and $70 \%$, respectively. In Figure 2.b, with more local Add/Drop requests ( $70 \%$ of all requests), BBP increases overall due to the transceiver scarcity and degree of (in)flexibility of all architectures except Flex-Tc2F\&C. Being the experienced BBP by Flex-Tc2F\&C exclusively due to transceiver scarcity, the extra BBP experienced by the remainder architectures can be attributed to their (in)flexibility. Flex-Tc2C seems to behave slightly better than Flex-Tp2F\&C here. And, again, Static-Tp becomes a simpler yet better option than Flex-Tp2C. In Figure 2.c, with $70 \%$ of bypass connections and only $30 \%$ of local Add/Drop ones, the performance of all architectures tend to be similar, which is also expectable. In fact, the same transceivers are available as in previous cases, but with less offered local Add/Drop requests.

\section{CONCLUSIONS}

This paper has analysed 5 different architectures for the Add/Drop module of future SDM-ROADMs, lowering hardware (i.e., equipment) complexity at expenses of inferior transceiver interconnection flexibility. Simulations in a single SDM-ROADM scenario show that the fully flexible Flex-Tc2F\&C architecture can yield the desired network performance $(\mathrm{BBP} \approx 1 \%$ ) with significantly less transceivers at the SDM-ROADM compared to the other architectures (30\% less compared to Flex-Tp2F\&C and Flex-Tc2C, and 45\% less compared to Static-Tp and Flex-Tp2C). It remains open for future work to investigate whether this transceiver reduction attainable by Flex-Tc2F\&C can pay off its added interconnection complexity and cost.

\section{ACKNOWLEDGEMENTS}

The work included in this paper has been supported by the Spanish Government through project ALLIANCE (TEC2017-90034-C2-1-R and TEC2017-90034-C2-2-R) with FEDER contribution. The work of M. Klinkowski was supported by National Science Centre, Poland under Grant 2016/21/B/ST7/02212.

\section{REFERENCES}

[1] G. M. Saridis, D. Alexandropoulos, G. Zervas, D. Simeonidou, "Survey and Evaluation of Space Division Multiplexing: From Technologies to Optical Networks," IEEE Commun. Surv. Tutor., vol. 17, no. 4, 2015.

[2] D. Klonidis, et al., "Spectrally and spatially flexible optical network planning and operations," IEEE Commun. Mag., vol. 53, no. 2, 2015.

[3] J. Perelló, J. M Gené, A. Pagès, J. A Lazaro, S. Spadaro, "Flex-grid/SDM backbone network design with inter-core XT-limited transmission reach," J. Opt. Commun. Netw., vol. 8, no. 8, 2016.

[4] B. Shariati, et al., "Physical-layer-aware performance evaluation of SDM networks based on SMF bundles, MCFs, and FMFs, " J. Opt. Commun. Netw., vol. 10, no. 9, 2018.

[5] B. Shariati, et al., "Impact of spatial and spectral granularity on the performance of SDM networks based on spatial superchannel switching," J. Light. Technol., vol. 35, no. 13, 2017.

[6] D. M. Marom, et al., "Survey of Photonic Switching Architectures and Technologies in Support of Spatially and Spectrally Flexible Optical Networking," J. Opt. Commun. Netw., vol. 9, no. 1, 2017.

[7] R. Rumipamba-Zambrano, F.-J. Moreno-Muro, J. Perelló, P. Pavón-Mariño, S. Spadaro, "Space Continuity Constraint in Dynamic Flex-Grid/SDM Optical Core Networks: An Evaluation with Spatial and Spectral Super-channels," Computer Communications, vol. 126, pp. 38-49, Aug. 2018. 